\title{
Investigation of a Cluster of 2019 Novel Coronavirus Disease (COVID-19) with Possible Transmission During the Incubation Period - Shenyang City, China, 2020
}

\author{
Ping Wang'; Zhiyong Lian'; Ye Chen'; Ying Q ${ }^{1}{ }^{1} ;$ Huijie Chen'; Xiangdong ${ }^{1} n^{1, *}$
}

\section{Background}

In December of 2019, a novel coronavirus (2019$\mathrm{nCoV}$ ) emerged in Wuhan China, which was later named COVID-19 (1). It quickly spread and as of February 11, 2020, there were 38,800 laboratoryconfirmed cases, 16,067 suspected cases, and 1,113 attributable deaths that have affected all provinciallevel administrative divisions (PLADs) of Mainland China (2).

The first confirmed COVID-19 case in Shenyang City, the capital of Liaoning Province, occurred on January 22, 2020, with a total of 9 confirmed cases by January 29, 2020. Of these cases, 4 were clustered in one family. Shenyang CDC immediately launched an investigation to determine routes of transmission, scale of the outbreak, and evidence for epidemic control.

\section{Methods and Case Finding}

This investigation focused on cases that were clustered. In-person interviews were conducted with a detailed questionnaire. Using the China CDC guideline definitions for suspected and confirmed COVID-19 cases, the following criteria was used.

A suspected case was defined in a person fulfilling at least 1 from items $1-4$ that revealed exposure history, 2 from items 5-7 that revealed clinical symptoms, or with items 5, 6, and 7 if there was no exposure history (3).

1. History of travel to or being a resident of Wuhan, areas surrounding Wuhan, or any other places where COVID-19 patients were reported.

2. Contact history with COVID-19 confirmed case(s).

3. Contact history with a patient with fever or respiratory symptoms who came from Wuhan, areas surrounding Wuhan, or other places where COVID19 patients were reported.

4 . Be one of the fever or respiratory symptom cluster cases.

5. Fever and/or respiratory symptoms.

6. Radiographic evidence of pneumonia.

7. Low or normal white-cell count or low lymphocyte count.

A confirmed case was defined as a suspected case with respiratory or blood specimens that tested positive for the COVID-19 by RT-PCR or with a genetic sequence highly homologous to COVID-19.

We did face to face interviews with all confirmed cases to understand their activities during the previous 14 days before onset of symptoms. All close contacts were advised to isolate themselves at home for 14 days with daily check-ins by local CDC staff and body temperature readings were required two times a day. If a contact developed fever or respiratory symptoms, they would immediately be sent to the hospital for testing by RT-PCR testing.

\section{Results}

History of the primary case:

January 17: A 21-year old student from Shenyang (Patient A) travelled to Wuhan with 5 classmates on January 17 and returned on January 20 by air while wearing masks. Her father picked her up at the airport at 3: $30 \mathrm{pm}$ via personal car, where both wore masks, to return home, and no one wore masks at home.

January 20 Evening: Patient A had dinner with her boyfriend, who later became Patient D, at 8 pm for 3 hours in a fast-food restaurant.

January 21: Patient A participated in a family dinner with seven other relatives including her father, mother, grandfather, grandmother, uncle, aunt, and cousin. All those at the dinner were later quarantined at home for 14 days. Besides her parents, the other five family members who attended the dinner showed no symptoms and their throat swabs tested negative for COVID-19 using RT-PCR.

January 22: Patient A had a party with 11 friends for 
6 hours including her boyfriend. They were later quarantined at home for 14 days.

January 23: Patient A became symptomatic around 4 $\mathrm{pm}$ with no previous symptoms. She then began having nasal congestion, runny nose, fatigue, headache, and fever $\left(37.3{ }^{\circ} \mathrm{C}\right)$. Her fever increased to $38.7{ }^{\circ} \mathrm{C}$ that night. Her father (Patient B) also had a fever that started at $6 \mathrm{pm}$ the same day.

January 24: Patient A and B went to the hospital together where both tested positive for COVID-19 by RT-PCR. They were diagnosed as confirmed cases and isolated at hospital.

January 25: The mother (Patient C) of Patient A was under self-isolation at home, experienced onset of fever, and was laboratory confirmed with COVID-19 on January 26.

January 27: Patient A's boyfriend experienced onset of fever and was confirmed with COVID-19 on January 28 . They only met twice at restaurant on 20 and 22, respectively. Among the four total cases in this cluster, only Patient A had traveled outside of Shenyang or had traveled to Wuhan, and none of the other three patients had traveled or had contact with another confirmed case (Table 1).

Among the 11 friends who had the party with patient $\mathrm{A}$, one friend, who sat next to patient $\mathrm{A}$ at the party, developed fever $\left(37.9{ }^{\circ} \mathrm{C}\right)$, sore throat and productive cough on Jan 25. However, her two successive throat swabs showed negative RT-PCR results for COVID-19. The other 10 friends showed no symptoms and their throat swabs all showed negative RT-PCR results for COVID-19 after 14 days quarantine.

Of the five other classmates that had traveled to Wuhan with Patient A, none developed symptoms during the 14 days of self-quarantine. Their throat swabs tested negative by RT-PCR for COVID-19. Everyone in the group who traveled to Wuhan bought duck snacks, a Hubei-Province delicacy, for their families and no further cases were found among those who ate the snacks. Figure 1 showed the epi curve of the 4 cases (Figure 1).

From the 4 cases, 43 contacts were identified, and all contacts were quarantined at home for 14 days and showed no symptoms. Patients were immediately isolated at the hospital when they were confirmed.

Among the 169 passengers and 14 crew who were on the flight returning from Wuhan to Shenyang with Patient A, another confirmed case of COVID-19 was found among the passengers. This passenger became febrile on January 25 and was COVID-19 confirmed on January 28 in Jiamusi, Heilongiiang Province. After arriving in Shenyang by air he traveled by train to Changchun on January 20. His 3 family members were infected in the several days following his return home. This passenger was seated 13 rows apart from Patient

TABLE 1. The cluster of COVID-19 cases by age, sex, onset of symptoms, laboratory confirmation, and travel history in Shenyang, China, 2020.

\begin{tabular}{|c|c|c|c|c|c|c|c|}
\hline Case & Sex & $\begin{array}{c}\text { Relationship to } \\
\text { patient } \mathrm{A}\end{array}$ & Age & $\begin{array}{c}\text { Onset of } \\
\text { symptoms }\end{array}$ & $\begin{array}{l}\text { Laboratory } \\
\text { confirmation }\end{array}$ & $\begin{array}{c}\text { Date of } \\
\text { diagnosis }\end{array}$ & Travel history \\
\hline$A$ & Female & Self & 21 & January 23 & Confirmed & January 24 & $\begin{array}{c}\text { Flight to Wuhan } 1 / 17 \\
\text { Return } 1 / 20\end{array}$ \\
\hline B & Male & Father & 44 & January 23 & Confirmed & January 24 & No travel \\
\hline $\mathrm{C}$ & Female & Mother & 45 & January 25 & Confirmed & January 26 & No travel \\
\hline $\mathrm{D}$ & Male & Boyfriend & 21 & January 27 & Confirmed & January 28 & No travel \\
\hline
\end{tabular}

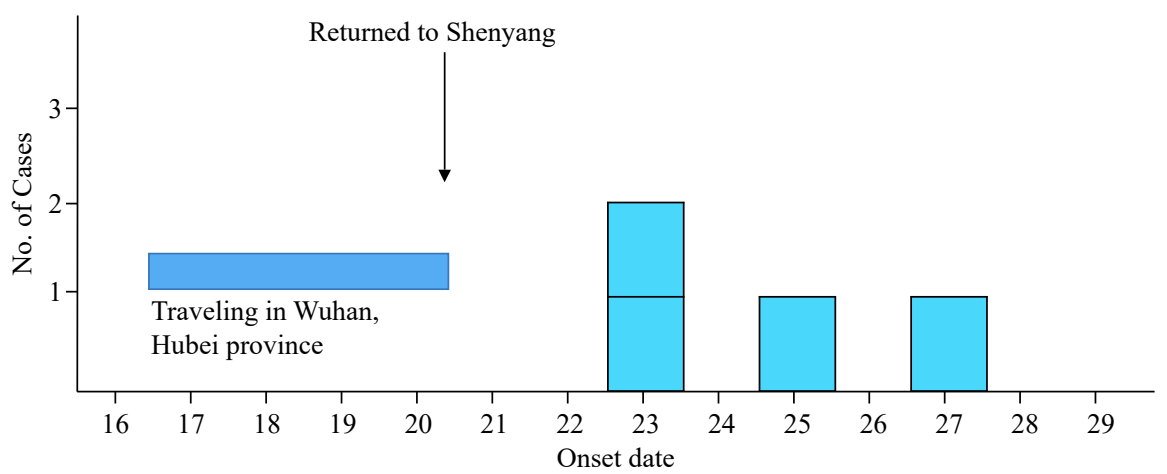

FIGURE 1. The epi curve of a cluster of COVID-19 cases of in Shenyang, China, 2020. 
A, did not know Patient A. The passenger said that he did not show any symptoms on the plane.

\section{Discussion}

Transmission of COVID-19 is thought to primarily be from patients with overt symptoms (coughing, sneezing, runny nose, fever, and contaminated hands). Spread of coronavirus during incubation period is considered quite possible given the transmission factors of other coronaviruses (4).

Based on the results of the cluster investigation, we concluded that Patient A likely transmitted COVID19 to 3 close contacts during her incubation period. Patient A was home 3 days before onset of symptoms, which occurred on the same day her father, Patient B, experienced onset of fever symptoms and the day before both were laboratory-confirmed with COVID19. Patient $C$, the mother, was exposed to Patient $A$ five days before onset of symptoms. Patient D, boyfriend, had symptoms five or seven days after exposure to Patient A. If COVID-19 can be transmitted in the incubation period, as possibly indicated in this investigation, then epidemic control will be more difficult (5).

Currently, close contact recommendations focus on those who had contact with cases after development of symptoms and 2 days before development of symptoms, which is in accordance with the national close contact guidelines for COVID-19 in China. More of an evidence base is needed to evaluate transmission during incubation period and its importance and contribution to the COVID-2019 epidemic.

\section{Acknowledgement}

We are grateful to Dr. Huilai Ma and Dr. Lijie Zhang (Chinese Field Epidemiology Training Program, China CDC) for their contributions in providing guidance to this investigation and thanks Dr. Linda Quick (US CDC) for her kind support to the manuscript and investigation.

\# Corresponding author: Xiangdong An, anjoyemail@126.com.

${ }^{1}$ Shenyang Center for Disease Control and Prevention, Shenyang, Liaoning, China.

Submitted: February 14, 2020; Accepted: February 19, 2020

\section{References}

1. Li Q, Guan XH, Wu P, Wang XY, Zhou L, Tong YQ, et al. Early transmission dynamics in Wuhan, China, of novel coronavirus-infected pneumonia. N Engl J Med 2000. http://dx.doi.org/10.1056/NEJMoa 2001316.

2. National Health Commission. Epidemic situation notificaiton. http:// www.nhc.gov.cn/xcs/yqtb/202002/395f075a5f3a411f80335766c65b048 7.shtml. (In Chinese). [2020-2-14].

3. General Office of National Health Commission. Prevention and control protocol for Novel Coronavirus Pneumonia (version 4). (2020-2-7) http://www.nhc.gov.cn/xcs/zhengcwj/202002/573340613ab243b3a7f61 df260551dd4.shtml. (In Chinese). [2020-2-14].

4. Al-Tawfiq JA, Auwaerter PG. Healthcare-associated infections: the hallmark of Middle East respiratory syndrome coronavirus with review of the literature. J Hosp Infect 2019;101(1):20 - 9. http://dx.doi.org/10. 1016/j.jhin.2018.05.021.

5. Callaway E, Cyranoski D. China coronavirus: six questions scientists are asking. Nature 2020;577(7792):605 - 7. http://dx.doi.org/10.1038/d415 86-020-00166-6. 